Artículo Original

\title{
Percepción de los estudiantes universitarios peruanos acerca de las repercusiones académicas generadas por la COVID-19
}

\section{Perception of peruvian university students about the academic repercussions generated by COVID-19}

https://doi.org/10.52808/bmsa.7e5.61e2.019

Victor Serna-Alarcón ${ }^{1 *}$

https://orcid.org/0000-0002-9803-6217

Karen M. Paraguay ${ }^{2}$

https://orcid.org/0000-0003-3904-9891

Arleth S. Mejía ${ }^{2}$

https://orcid.org/0000-0002-0834-003X

J. Franco Rodriguez-Alarcón ${ }^{3,4}$

https://orcid.org/0000-0003-4059-8214

Daniella Vinelli-Arzubiaga ${ }^{3}$

https://orcid.org/0000-0002-8793-2446

Martin A. Vilela-Estrada ${ }^{1}$

https://orcid.org/0000-0002-1494-952X

Lipselotte de Jesús Infante Rivera ${ }^{2}$

https://orcid.org/0000-0001-6094-1070

Christian R. Mejia ${ }^{5}$

Recibido: 07/04/2021

https://orcid.org/0000-0002-5940-7281

Aceptado: 28/07/202

\section{RESUMEN}

El objetivo principal de la investigación fue tratar de ver la percepción de las clases virtuales y las posibles repercusiones académicas que tuviesen estudiantes universitarios en el Perú, y sus factores asociados a los mismos, durante la pandemia de la COVID-19. Estudio transversal analítico, en estudiantes universitarios del Perú durante la primera ola de la pandemia, la variable que media la percepción se obtuvo a través de una escala validada. En la estadística analítica, se encontró que las mujeres tuvieron una mayor frecuencia de percepción de repercusiones académicas (RPa: 1,37; IC95\%: 1,18-1,59; valor p<0,001), esto ajustado por la edad, la carrera de estudios y la universidad. Las mujeres fueron las que percibían más repercusiones. Si bien solo se encontró asociación con el sexo de los encuestados, se deberían realizar estudios más grandes y en etapas más avanzadas de la pandemia, ya que, nuestros resultados se pueden tomar como basales, pero debería hacerse el seguimiento y determinar la influencia de otras variables.

Palabras clave: COVID-19, repercusión académica, coronavirus, Perú, estudiantes.

\section{ABSTRACT}

The main objective of the research was to try to see the perception of virtual classes and the possible academic repercussions that university students had in Peru, and their associated factors, during the COVID-19 pandemic. Analytical cross-sectional study in Peruvian university students during the first wave of the pandemic, the variable that mediates perception was obtained through a validated scale. In the analytical statistics, it was found that women had a higher frequency of perception of academic repercussions (PRa: 1.37; 95\% CI: 1.18-1.59; $p$ value <0.001), this adjusted for age, study career and university. Women were the ones who perceived the most repercussions. Although an association was only found with the sex of the respondents, larger studies should be carried out and in more advanced stages of the pandemic, since our results can be taken as baseline, but the influence of others should be followed up and determine the influence of other variables.

Key words: COVID-19, academic impact, coronavirus, Peru, students.

1. Escuela de Medicina Humana, Universidad Privada Antenor Orrego. Trujillo, Perú 2. Universidad Continental. Huancayo, Perú. 3. Asociación Médica de Investigación y Servicios en Salud Lima, Perú. 4. Facultad de Medicina Humana "Manuel Huamán Guerrero”. Universidad Ricardo Palma. Lima, Perú. 5. Centro de Investigación en Medicina Traslacional. Universidad Norbert Wiener. Lima, Perú.

*Autor de Correspondencia: vsernaa@upao.edu.pe

\section{Introducción}

La contingencia sanitaria generada por el COVID-19 ocasionó diversos cambios a nivel de todas las instancias de la sociedad, y por ende, las instituciones educativas no estaban preparadas para estos cambios (Gazca Herrera, 2020). 
Esto debido a las medidas gubernamentales tomadas por las autoridades a cargo de cada país, que establecieron diversas medidas restrictivas, como el permanecer por una gran cantidad de meses en nuestros hogares, aislamiento social, toques de queda, restricción para la movilización, entre otras muchas (Iglesias-Osores, 2020; Zhao, 2020). Lo que también implicó que la enseñanza cambiase de rumbo, ya que, la gran mayoría de entidades educativas tenían como base la educación presencial, esto a pesar de que algunas pocas universidades o instituciones ya habían dado el salto a la educación virtual. Por lo tanto, los alumnos, docentes y el personal administrativo de las instituciones educativas, tuvieron que adaptarse a esta nueva modalidad educativa, y todo de forma repentina (López et al., 2021; MartínezGarcés et al., 2020).

Lo cual repercutió de distinta manera, por ejemplo, las clases teóricas fueron las menos afectadas por el hecho de que solo se requiere un interlocutor y se imparte a estudiantes que estén dispuestos a recibir las clases "magistrales" o clásicas (Tapia Salinas et al., 2020). Sin embargo, las clases prácticas de las carreras que tienen sentido más empírico fueron tremendamente afectadas, debido a que estas son muy difíciles de realizar de manera virtual (Torda, 2020). Lo que generó que los estudiantes percibieran incertidumbre, temor y miedo, debido a que esto podría repercutir en su desempeño académico, en su captación del conocimiento y otros muchos aspectos con respecto a su futuro profesional (Rose, 2020).

Entonces, resulta necesario que esto se evalúe en una amplia población afectada durante los primeros meses de la pandemia, lo que mostraría un análisis basal de lo que pasó cuando recién se iniciaban las clases virtuales y mientras se percibían las primeras repercusiones. Si bien existen algunos reportes en otras realidades (España), en el Perú no hay muchos estudios que hayan abarcado este tema. Es por todo esto que el objetivo principal de la investigación fue determinar la percepción de las clases virtuales y las posibles repercusiones académicas que tuviesen los estudiantes universitarios en el Perú, y sus factores asociados a los mismos, durante la pandemia de la COVID-19.

\section{Materiales y Métodos}

Se realizó una investigación de tipo transversal (ya que a cada encuestado se le evaluó una sola vez) y analítica (al tener estadísticas descriptivas y analíticas en los resultados). Esto se ejecutó en las ciudades más importantes de todo el Perú (se tienen a las más grandes ciudades: Lima, Arequipa, Trujillo, Piura, Huancayo) y a otras ciudades de mediana y pequeña cantidad de habitantes (Ayacucho, Cajamarca, Chiclayo, Chimbote, Cusco, Huánuco, Ica, Iquitos, Cerro de Pasco, Puno y Tacna).

Se incluyó a los estudiantes de diversas facultades y carreras que durante la primera ola del coronavirus estuviesen matriculados o a la espera del inicio de clases durante los meses de mayo y junio del 2020 (esto debido a que algunos ya habían empezado las clases y otras universidades todavía estaban a la espera de mejorar sus procesos virtuales para poder tener clases a distancia). Se excluyó aquellos estudiantes que no respondiesen la totalidad de las preguntas del test de repercusiones académicas, ya que, sin estas preguntas totales no se podría tener la variable principal del estudio. Además, también se excluyó a los que dieran respuestas incompletas en la mayoría de las preguntas o a los que tuviesen respuestas anómalas (todas las exclusiones llegaron a ser menos del 10\% de la muestra total).

Se llegó a calcular la potencia estadística del cruce de cada una de las percepciones de repercusión académica según las variables obtenidas, sabiendo que se debía tener valores mayores al $80 \%$ para que un cruce sea significativo en su potencia estadística. Sabiendo que la totalidad de las encuestas se obtuvo a través de un muestreo por conveniencia.

La variable principal fue la percepción de la repercusión académica que tenían los estudiantes encuestados, esto fue realizado a través de la escala ACAD-COVID 19 (Mejia et al., 2021), que fue validada en la población peruana estudiantil (la escala de ACAD-COVID-19 mide la percepción de los estudiantes sobre el impacto académico que han sido generadas por el coronavirus); es importante mencionar que las preguntas están desarrolladas de forma que las pudiesen responder aquellos que ya habían iniciado las clases o los que aún estaban en suspensión de actividades académicas. Esta escala ha demostrado que tiene una buena validez interna, obteniéndose un alfa de Cronbach del 0,899; con un intervalo de confianza del $95 \%$ entre 0,882 a 0,898. Además, ha obtenido muy buenos puntajes en cuanto a la V de Aiken (>0,70), a los valores del Kaiser-Meyer-Olkin (KMO) $(0,85)$, la prueba de Bartlett $(\mathrm{p}<0,001)$ y otros, que en su conjunto muestran una adecuada consistencia en los ítems utilizados. La escala final cuenta con 8 ítems, que miden de forma directa cada uno de los miedos o preocupaciones que tienen los estudiantes encuestados acerca de las dificultades, problemas u otras percepciones de las repercusiones que puede tener el llevar las clases de manera virtual o a distancia.

Además se midió algunas otras variables que puedan ser importantes para la caracterización de la población, como lo son el sexo del encuestado, la edad que manifestó hasta el momento de la encuesta, el tipo de la universidad en la que estudiaba (particular o estatal), el departamento en la cual residía (dentro de los departamentos ya mencionados), 
el tipo de carrera que estaba realizando (7 posibles carreras) y el año académico en que se encontraba (tomado según el año académico que estaba realizando o en el que se quedó).

La investigación se basa en la data ya recolectada entre los meses de mayo y junio a una población estudiantil de miles de encuestados en todo el Perú. Esto se realizó a través de un encuestado virtual, para lo cual se usó la herramienta Formularios de Google. Debido a que así fue aprobado por el comité de ética inicial (Universidad Privada Antenor Orrego), además, esto también por el hecho de que en esos meses aun el Perú se encontraba en la primera ola de la enfermedad de la COVID-19, por lo que, existían múltiples medidas de restricción y confinamiento (haciendo imposible el hacer encuestas de manera presencial). Una vez que se obtuvo la base de datos se depuró para poder quedarse con la población que cumpliese los criterios de selección, aquí es cuando se realizó el control de calidad (para poder encontrar discrepancias y poder eliminar las encuestas que no sean coherentes) y luego se realizó la exportación a un programa estadístico (al programa Stata en su versión 11,1).

Primero se describió a la población a través de sus características, teniendo las variables categóricas y cuantitativas. Se describió las primeras a través de sus frecuencias y porcentajes, a las variables cuantitativas se le encontraron la mejor medida de tendencias central y dispersión. Luego se realizó un análisis bivariado, en donde se pudo obtener la potencia estadística y el análisis crudo de las variables. En el tercer paso se consideró a las variables que hayan obtenido un valor significativo ( $\mathrm{p}<0,05)$, a las que se las cruzó en un modelo multivariado. En los últimos pasos se utilizó los modelos lineales generalizados, con la familia Poisson, la función de enlace log, modelos para varianzas robustas y usando como grupo "cluster" a la universidad en donde estudiaba; con los cuales se obtuvo las razones de prevalencia, los intervalos de confianza y los valores p. Cabe resaltar que todos estos pasos se consideró un nivel de confianza del $95 \%$ y la significancia estadística tuvo el punto de corte de 0,05.

\section{Resultados}

De los 2138 estudiantes encuestados, las principales preocupaciones que tenían eran acerca de perder el ciclo ( $31 \%$ muy de acuerdo y $35 \%$ de acuerdo) y de perder todo el año académico (31\% muy de acuerdo y $34 \%$ de acuerdo), esto seguido de la preocupación de no saber cómo terminaría el año académico (30\% muy de acuerdo y $42 \%$ de acuerdo) (Tabla 1).

Tabla 1. Porcentajes de las percepciones de repercusión académica que tenían los estudiantes de educación superior durante la primera ola del COVID-19 en el Perú

\begin{tabular}{|c|c|c|c|c|c|}
\hline $\begin{array}{l}\text { Percepción de las posibles repercusiones académicas que puedan } \\
\text { tener los estudiantes }\end{array}$ & $\begin{array}{c}\text { Muy en } \\
\text { desacuerdo }\end{array}$ & $\begin{array}{c}\text { En } \\
\text { desacuerdo }\end{array}$ & Indiferente & $\begin{array}{c}\text { De } \\
\text { acuerdo }\end{array}$ & $\begin{array}{l}\text { Muy de } \\
\text { acuerdo }\end{array}$ \\
\hline $\begin{array}{l}\text { Tengo miedo/ preocupación de no adaptarme al posible acelerado ritmo } \\
\text { académico }\end{array}$ & $11 \%$ & $13 \%$ & $16 \%$ & $37 \%$ & $23 \%$ \\
\hline $\begin{array}{l}\text { Tengo miedo /preocupación de bajar mi promedio ponderado tras el } \\
\text { retorno a clases. }\end{array}$ & $11 \%$ & $16 \%$ & $19 \%$ & $34 \%$ & $20 \%$ \\
\hline Tengo miedo/preocupación de perder el ciclo & $9 \%$ & $12 \%$ & $13 \%$ & $35 \%$ & $31 \%$ \\
\hline Tengo miedo/preocupación por perder el año académico & $9 \%$ & $12 \%$ & $14 \%$ & $34 \%$ & $31 \%$ \\
\hline $\begin{array}{l}\text { Tengo miedo/preocupación de exponerme al COVID-19 tras retornar a } \\
\text { clases }\end{array}$ & $8 \%$ & $11 \%$ & $16 \%$ & $39 \%$ & $26 \%$ \\
\hline Tengo miedo/preocupación de morir por el COVID-19 & $19 \%$ & $19 \%$ & $23 \%$ & $23 \%$ & $16 \%$ \\
\hline $\begin{array}{l}\text { Tengo preocupación por enfrentarme a una desestabilidad económica } \\
\text { familiar y no poder pagar mi ciclo académico }\end{array}$ & $10 \%$ & $14 \%$ & $15 \%$ & $36 \%$ & $25 \%$ \\
\hline Tengo preocupación de no saber cómo terminará el año académico & $6 \%$ & $9 \%$ & $13 \%$ & $42 \%$ & $30 \%$ \\
\hline
\end{tabular}

Al sumar los puntajes de cada una de las 8 preguntas, esto se cruzó versus el año de estudios, obteniendo que los de primer año tenían una mediana de más de 30 puntos, los de segundo y tercer año una mediana de 30 puntos, y así sucesivamente fueron bajando las medianas; lo que muestra que la percepción de preocupación disminuyó conforme aumentaba el año de estudios (Figura 1). 


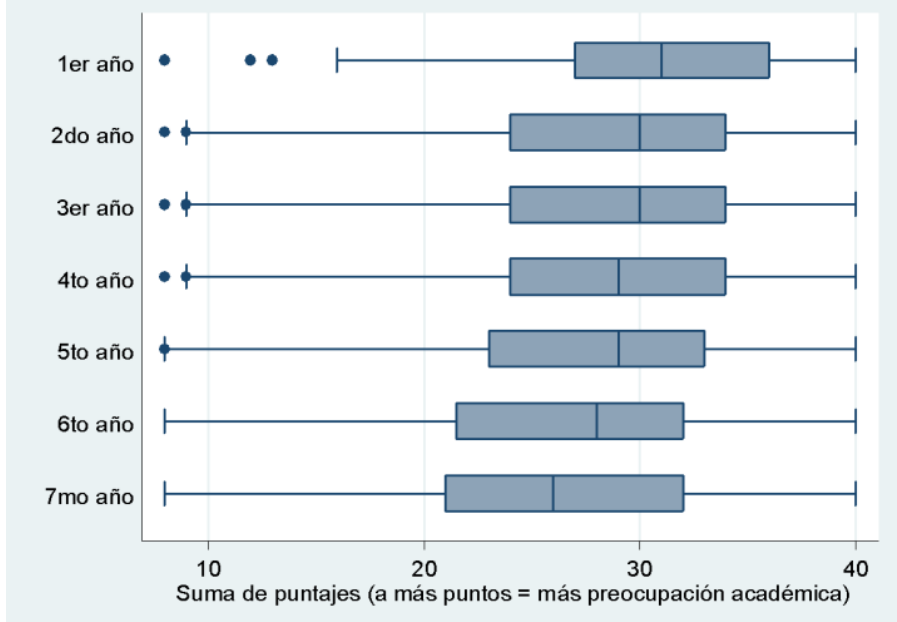

Figura 1. Gráfico de cajas y bigotes de la suma de puntajes de la percepción de repercusión académica versus el año de estudios en educación superior durante la primera ola del COVID-19 en el Perú

Cuando se realizó el análisis bivariado de los factores asociados a la percepción de repercusiones académicas, se halló que hubo asociación con el sexo $(\mathrm{p}<0,001)$ y la edad $(\mathrm{p}<0,001)$, no se encontró asociación con la carrera y si el estudiante era migrante (Tabla 2).

Tabla 2. Factores asociados a la percepción de repercusiones académicas en alumnos de educación superior durante la primera ola del COVID-19 en el Perú

\begin{tabular}{lccc}
\hline \multirow{2}{*}{ Variables } & \multicolumn{2}{c}{ Perciben repercusión académica } & \multirow{2}{*}{ Valor p } \\
\cline { 2 - 3 } & \multicolumn{1}{c}{ No } & Si & \\
\hline Sexo & $667(68,3 \%)$ & $309(31,7 \%)$ & $<0,001$ \\
$\quad$ Masculino & $660(56,8 \%)$ & $502(43,2 \%)$ & \\
Femenino & $22(20-23)$ & $21(20-23)$ & $<0,001$ \\
Edad (años)* & & & \\
Carrera & $818(64,1 \%)$ & $458(35,9 \%)$ & \\
$\quad$ Salud & $162(60,0 \%)$ & $108(40,0 \%)$ & \\
Ingeniería & $49(62,0 \%)$ & $30(38,0 \%)$ & \\
Derecho & $87(57,2 \%)$ & $65(42,8 \%)$ & \\
Administración & $40(55,6 \%)$ & $32(44,4 \%)$ & \\
Humanidades & $18(56,3 \%)$ & $14(43,7 \%)$ & \\
Educación & $86(58,1 \%)$ & $62(41,9 \%)$ & \\
Ciencias básicas & $44(62,0 \%)$ & $27(38,0 \%)$ & \\
Sociales & $23(60,5 \%)$ & $15(39,5 \%)$ & 0,533 \\
Artes & & & \\
El estudiante vive & $887(63,2 \%)$ & $517(36,8 \%)$ & \\
$\quad$ En la misma ciudad & $440(60,0 \%)$ & $294(40,0 \%)$ & 0,144 \\
$\quad$ Es migrante & & & \\
\hline
\end{tabular}

*Se muestran resultados descriptivos de las medianas y los rangos intercuartílicos. Los valores p fueron obtenidos con la suma de rangos (para la edad) y el chi cuadrado (para los demás cruces).

En la estadística analítica, se encontró que las mujeres tuvieron una mayor frecuencia de percepción de repercusiones académicas ( $\mathrm{RPa}$ : 1,37; IC95\%: 1,18-1,59; valor $\mathrm{p}<0,001$ ), esto ajustado por la edad, la carrera de estudios y la universidad (Tabla 3 ).

\section{Discusión}

Las principales preocupaciones que tenían los estudiantes universitarios eran perder el ciclo o el año académico, así como, la preocupación por no saber cómo terminaría el año académico. Con la llegada de la pandemia de la COVID-19 muchas instituciones educativas tuvieron que adecuarse al sistema virtual, impidiendo la presencialidad en sus aulas, lo que afectó a miles de estudiantes y causó preocupación de los mismos (UNESCOIESALC, 2020). Esta nueva adaptación al sistema virtual se convirtió en muchos casos en un reto, tanto para los alumnos como para los docentes, quienes antes no habían tenido la necesidad de usar estas plataformas (MartínezGarcés \& Garcés-Fuenmayor, 2020). A todo esto, se sumó las desigualdades en cuanto a la accesibilidad a una buena y eficiente señal, ya sea por una dificultad geográfica, económica o simplemente por no contaban con el implemento, 
básico que es la computadora de uso personal y la red que surtía de la adecuada señal (Quintana Avello, 2020). Además, la pandemia trajo también consigo repercusiones en el ámbito económico, siendo una de las preocupaciones de los estudiantes el enfrentarse a una desestabilidad económica familiar y no poder pagar el ciclo académico (esto en las universidades particulares a través de la dificultad para el pago de las pensiones mensuales hacia la universidad, así como, en las nacionales los gastos iban relacionados a aspectos de mantención de la estabilidad económica familiar), esto se ha evidenciado en el aumento de la deserción académica durante los primeros meses de la pandemia en países como Colombia (Rincon et al., 2020) y Perú (Lovón Cueva \& Cisneros Terrones, 2020).

Tabla 3. Estadística bivariada y multivariada de los factores asociados a la percepción de repercusiones académicas en alumnos de educación superior durante la primera ola del COVID-19 en el Perú.

\begin{tabular}{|c|c|c|}
\hline Variables & Bivariado & Multivariado \\
\hline \multicolumn{3}{|l|}{ Sexo } \\
\hline Masculino & Ref. & Ref. \\
\hline Femenino & $1,36(1,19-1,57)<0,001$ & $1,37(1,18-1,59)<0,001$ \\
\hline Edad (años)* & $0,96(0,93-0,99) 0,034$ & $0,97(0,93-1,00) 0,065$ \\
\hline \multicolumn{3}{|l|}{ Carrera } \\
\hline Salud & Ref. & Ref. \\
\hline Ingeniería & $1,11(0,91-1,36) 0,283$ & $1,21(0,99-1,48) 0,069$ \\
\hline Derecho & $1,06(0,71-1,57) 0,781$ & $1,03(0,71-1,49) 0,877$ \\
\hline Administración & $1,19(0,95-1,49) 0,127$ & $1,20(0,97-1,48) 0,089$ \\
\hline Humanidades & $1,24(1,00-1,53) 0,049$ & $1,21(0,98-1,48) 0,070$ \\
\hline Educación & $1,22(0,79-1,89) 0,374$ & $1,15(0,75-1,74) 0,527$ \\
\hline Ciencias básicas & $1,17(0,94-1,45) 0,157$ & $1,19(0,97-1,46) 0,098$ \\
\hline Sociales & $1,06(0,79-1,41) 0,695$ & $0,99(0,75-1,33) 0,983$ \\
\hline Artes & $1,10(0,75-1,62) 0,630$ & $1,10(0,75-1,61) 0,621$ \\
\hline \multicolumn{3}{|l|}{ El estudiante vive } \\
\hline En la misma ciudad & Ref. & No entró al modelo \\
\hline Es migrante & $1,09(0,96-1,23) 0,192$ & No entró al modelo \\
\hline
\end{tabular}

Otra de las preocupaciones de los estudiantes fue el exponerse al COVID-19 tras retornar a clases presenciales y el miedo a morir por esta nueva enfermedad. Si bien el regreso a las clases se dará progresivamente, cumpliendo las medidas de bioseguridad y cuando los docentes y estudiantes estén vacunados, aun así, genera incertidumbre de cómo sería en ese momento. Además, es necesario recordar que los estudiantes de ciencias de la salud aún se van a encontrar expuestos a este nuevo virus y a sus variaciones, siendo una población en mayor riesgo por el ambiente en el que se desempeñan (Khamees et al., 2020). Un estudio realizado en estudiantes de enfermería encontró que, si bien ellos reconocían su responsabilidad con su comunidad y los riesgos de su profesión, la mayoría tenía miedo de un posible contagio, sobre todo en los entornos clínicos (Lovrić et al., 2020). Entonces, es necesario que las instituciones educativas y los centros de salud garanticen la seguridad de sus estudiantes, priorizando en este grupo la vacunación antes del regreso a sus prácticas clínicas.

También se encontró en la estadística descriptiva que la percepción de preocupación disminuyó conforme aumentaba el año de estudios, esto podría deberse a que lo estudiantes de primeros ciclos concluyen la secundaria e ingresaban a un entorno nuevo, donde se tienen que adaptar a una nueva institución, nuevos compañeros, al trabajo escolar y a los nuevos profesores, e incluso en muchos casos, se rompe el mundo familiar y escolar previo (Silva Laya, 2011). Además, un joven en los primeros años en la universidad aún se está adaptando a la vida universitaria, a diferencia de los que cursan ciclos superiores. De igual manera, otros autores mencionan que los estudiantes del primer año son poco maduros, llegando a presentar actitudes propias de la edad, en comparación con los estudiantes de últimos años, que presentan una madurez y responsabilidad superior (Rodríguez Espinar, 2015).

Por otro lado, fueron las mujeres quienes tuvieron una mayor percepción de las repercusiones académicas. Resultados similares se han evidenciado en otro estudio realizado en estudiantes universitarios, donde más del $70 \%$ de mujeres tenían niveles altos de miedo, estrés, ansiedad y depresión frente a las medidas de encierro que tomó el gobierno frente a la pandemia de la COVID-19 (Rodríguez-Hidalgo et al., 2020). De igual manera, un estudio realizado en Turquía (en 506 estudiantes de pregrado) asocia al género femenino positivamente con el miedo frente al estrés frente a la COVID-19 (Yalçın et al., 2021). Entonces queda en evidencia que las mujeres son las que más tendencias al miedo presentan, lo que podría influir en la percepción de las repercusiones académicas que tienen. Además, otro estudio menciona que las mujeres buscan tener mayor autoeficacia académica y mayores deseos de culminar sus estudios con éxito, en comparación con los hombres (Flores e et al., 2014), esto es importante pues las expectativas de autoeficacia podrían ser elementos determinantes en las percepciones académicas. 
En este estudio la diferencia según la carrera estudiada no fue estadísticamente significativa, pero se encontró que los estudiantes de ciencias de la salud son los que generalmente se reconocen como una población de riesgo para padecer ansiedad y depresión en todo el mundo, esto se demostró en diferentes estudios (Garcia-Espinosa et al., 2021). En un estudio realizado en China el $25 \%$ presentaron ansiedad relacionada a la pandemia, siendo el principal factor el miedo al retraso en educación y el factor económico (Cao et al., 2020). Por lo tanto, esta asociación encontrada debe ser evaluada en futuras investigaciones que intenten determinar si los estudiantes de ciencias de la salud son los que tienen una mayor percepción de las repercusiones académicas, lo que permitiría que se desarrollen estrategias de intervención en los centros universitarios para el cuidado de la salud mental de los estudiantes según su carrera universitaria.

El estudio presentó algunas limitaciones, siendo la más importante el sesgo de selección, en el que se incurrió debido a que no se realizó un muestreo aleatorio; lo que no permite poder extrapolar los resultados a la totalidad de estudiantes en todos los departamentos o en las universidades de donde provenían. Sin embargo, esta solo fue una limitación en parte, ya que, nunca se intentó tratar de extrapolar los resultados a la población global de las universidades o departamentos, lo que sí se quiso hacer desde el inicio fue extrapolar los resultados para conseguir las asociaciones (esto se logró gracias a tener una población de 2138 estudiantes encuestados). También hubiese sido importante tener la presencia de otras variables que pudiesen haber aportado al estudio, sin embargo, esto se espera que se realice en futuras investigaciones que se generen en cada universidad o que las dirijan las instituciones gubernamentales de cada país, para delimitar las posibles repercusiones que perciben los estudiantes por todo este incidente de la COVID-19.

Debido a todo lo encontrado, se concluye que las mujeres tuvieron una mayor frecuencia de percepción de las repercusiones académicas, esto ajustado por las variables. No hubo evidencia estadísticamente significativa de las repercusiones académicas según la edad, la carrera o el lugar de residencia de los estudiantes. También se encontró importantes miedos y preocupaciones en los estudiantes con respecto a algunas premisas de las posibles repercusiones que tendrían. Se recomienda que se realicen estudios de seguimiento, para determinar cómo evolucionó esta percepción según momentos diferentes de la pandemia (ya que nuestros resultados se dan en los momentos iniciales de la misma), además, que se evalúe si es que dichas percepciones se llegaron a cumplir (y en qué aspectos, cursos y casas de estudios).

\section{Conflicto de intereses}

Los autores declaran no tener conflicto de interés.

\section{Agradecimientos}

A la red de investigación GIS-COVID-19-Peru; que fue la coordinadora de la investigación primaria, así como, a cada uno de sus miembros, que apoyaron en la recolección inicial de la data.

\section{Referencias}

Cao, W., Fang, Z., Hou, G., Han, M., Xu, X., Dong, J., \& Zheng, J. (2020). The psychological impact of the COVID-19 epidemic on college students in China. Psychiatry Research, 287(112934):1-5. https://doi.org/10.1016/j.psychres.2020.112934

Flores, F. J., Mayorga-Vega, D., Blanco, J. R., \& Blanco, H. (2014). Perceived Self-Efficacy in Problem Solving and Scientific Communication in University Students. A Gender Study. Psychology, 2014(5):1-7. https://doi.org/10.4236/psych.2014.55046

Garcia-Espinosa, P., Ortiz-Jiménez, X., Botello-Hernández, E., Hernández-Díaz, A., Góngora-Rivera, F., \& Investigators, G. (2021). Covid-19, impacto psicosocial en estudiantes de medicina. Salud Pública de México, 63(3):330-331. https://doi.org/10.21149/12300

Gazca Herrera, L. A. (2020). Implicaciones del coronavirus covid-19 en los procesos de enseñanza en la educación superior. RIDE. Revista Iberoamericana para la Investigación y el Desarrollo Educativo, 11(21):e133. https://doi.org/10.23913/ride.v11i21.753

Iglesias-Osores, S. (2020). Importancia del aislamiento social en la pandemia de la COVID-19. Revista Medica Herediana, 31(3):205-206. https://doi.org/10.20453/rmh.v31i3.3814

Khamees, D., Brown, C. A., Arribas, M., Murphey, A. C., Haas, M. R. C., \& House, J. B. (2020). In Crisis: Medical Students in the COVID-19 Pandemic. AEM Education and Training, 4(3):284-290. https://doi.org/10.1002/aet2.10450 
López, E. M. P., Atochero, A. V., \& Rivero, S. C. (2021). Educación a distancia en tiempos de COVID-19: Análisis desde la perspectiva de los estudiantes universitarios. RIED: revista iberoamericana de educación a distancia, 24(1):331-350. https://doi.org/10.5944/ried.24.1.27855

Lovón Cueva, M. A., \& Cisneros Terrones, S. A. (2020). Repercusiones de las clases virtuales en los estudiantes universitarios en el contexto de la cuarentena por COVID-19: El caso de la PUCP. Propósitos y Representaciones, 8(SPE3):588. https://doi.org/10.20511/pyr2020.v8nSPE3.588

Lovrić, R., Farčić, N., Mikšić, Š., \& Včev, A. (2020). Studying During the COVID-19 Pandemic: A Qualitative Inductive Content Analysis of Nursing Students' Perceptions and Experiences. Education Sciences, 10(7):188. https://doi.org/10.3390/educsci10070188

Martínez-Garcés, J., \& Garcés-Fuenmayor, J. (2020). Competencias digitales docentes y el reto de la educación virtual derivado de la covid-19. Educación y Humanismo, 22(39):1-16. https://doi.org/10.17081/eduhum.22.39.4114

Mejia, C. R., Rodriguez-Alarcon, J. F., Charri, J. C., Liendo-Venegas, D., Morocho-Alburqueque, N., Benites-Ibarra, C. A., Avalos-Reyes, M. S., Medina-Palomino, D. S., Carranza-Esteban, R. F., Mamani-Benito, O., Mejia, C. R., Rodriguez-Alarcon, J. F., Charri, J. C., Liendo-Venegas, D., Morocho-Alburqueque, N., Benites-Ibarra, C. A., Avalos-Reyes, M. S., Medina-Palomino, D. S., Carranza-Esteban, R. F., \& Mamani-Benito, O. (2021). Repercusión académica de la COVID-19 en universitarios peruanos. Revista Cubana de Investigaciones Biomédicas, 40(1):e814. Disponible en: http://www.revibiomedica.sld.cu/index.php/ibi/rt/captureCite/814/0 (Acceso narzo 2021)

Quintana Avello, I. (2020). Covid-19 y Cierre de Universidades ¿Preparados para una Educación a Distancia de Calidad?. Revista Internacional De Educación Para La Justicia Social, 9(3). Disponoble en: https://revistas.uam.es/riejs/article/view/12232 (Acceso marzo 2021)

Rincon, I., Suarez, S., \& Suarez, A. (2020). Impacto del programa Jóvenes en Acción en la deserción estudiantil en tiempos de Covid-19. Espacios, 41(42):304-315. https://doi.org/10.48082/espacios-a20v41n42p25

Rodríguez Espinar, S. (2015). Los estudiantes universitarios de hoy: Una visión multinivel. Revista de Docencia Universitaria, 13(2):91-124. https://doi.org/10.4995/redu.2015.5440

Rodríguez-Hidalgo, A. J., Pantaleón, Y., Dios, I., \& Falla, D. (2020). Fear of COVID-19, Stress, and Anxiety in University Undergraduate Students: A Predictive Model for Depression. Frontiers in Psychology, 11(591797):1-9. https://doi.org/10.3389/fpsyg.2020.591797

Rose, S. (2020). Medical Student Education in the Time of COVID-19. JAMA, 323(21):2131-2132. https://doi.org/10.1001/jama.2020.5227

Sánchez Mendiola, M., Martínez Hernández, A. M. del P., Torres Carrasco, R., de Agüero Servín, M., Hernández Romo, A. K., Benavides Lara, M. A., Rendón González, V. J., \& Jaimes Vergara, C. A. (2020). Retos educativos durante la pandemia de COVID-19: Una encuesta a profesores de la UNAM. Revista Digital Universitaria, 21(3):1-23. https://doi.org/10.22201/codeic.16076079e.2020.v21n3.a12

Silva Laya, M. (2011). El primer año universitario: Un tramo crítico para el éxito académico. Perfiles educativos, 33(spe):102-114. Disponoble en: https://www.redalyc.org/pdf/132/13221258010.pdf (Acceso narzo 2021)

Tapia Salinas, J. A., Faican Yacuma, J. A., Checa de la Cruz, D. N., Quinche Martínez, F. R., \& Chicaiza Ñaunay, T. R. (2020). La educación virtual en tiempos de pandemia. Revista Cognosis. ISSN 2588-0578, 5(3):57-68. https://doi.org/10.33936/cognosis.v5i3.2493

Torda, A. (2020). How COVID-19 has pushed us into a medical education revolution. Internal Medicine Journal, 50(9): 1150-1153. https://doi.org/10.1111/imj.14882

UNESCO-IESALC. (2020). COVID-19 y educación superior: De los efectos inmediatos al día después (N.o 1; COVID19 Respuesta, pp. 1-57). Organización de las Naciones Unidas para la Educación, la Ciencia y la Cultura. Disponible en: https://www.iesalc.unesco.org/wp-content/uploads/2020/05/COVID-19-ES-130520.pdf (Acceso febrero 2021)

Yalçın, İ., Can, N., Mançe Çalışır, Ö., Yalçın, S., \& Çolak, B. (2021). Latent profile analysis of COVID-19 fear, depression, anxiety, stress, mindfulness, and resilience. Current Psychology (New Brunswick, N.J.), 1-11. https://doi.org/10.1007/s12144-021-01667-x

Zhao, G. (2020). Tomar medidas preventivas inmediatamente: Evidencia de China sobre el COVID-19. Gaceta Sanitaria, 34(1):217-219. https://doi.org/10.1016/j.gaceta.2020.03.002 